\title{
Pseudohypoparathyroidism: A Rare but Important Cause of Hypocalcaemia A Case Report
}

\author{
${ }^{1}$ Dr Duyu Hanang, ${ }^{2}$ Dr.Kamlom Mossang, ${ }^{3}$ Prof. Lallan Prasad, \\ ${ }^{4}$ Prof. Shanta Naorem, Associate ${ }^{5}$ Prof. Ranabir Salam, \\ ${ }^{6}$ Dr. E.Chumdemo Kikon \\ Regional Institute Of Medical Sciences
}

\section{Introduction}

Pseudohypoparathyroidism (PHP) is a rare heterogeneous group of disorder characterised by an endorgan resistance to parathyroid harmone (PHP) especially in kidney and bones, in which other hormonal deficiencies such as hypothyroidism and hypogonadism may coexist. ${ }^{1}$ A Japanese study estimated the prevalence of the disorder to be 7.2 per million only. ${ }^{2}$ Pseudohypoparathyroidism is a clinically dysmorphic syndrome characterised by developmental and skeletal defects that include short stature, rounded face, shortened fourth metacarpals and other bones of the hands and feet, obesity ,dental hypoplasia, soft tissue calcification/ossifications mostly in basal ganglia of brain. Although the features are characteristic they may vary, as many of the patients usually show unremarkable phenotypic features.

\section{History and Examination}

A 15 years old male was admitted with history of headache on and off since last 6 months. On depth clinical systemic examination there was occasional muscle cramps, episodic twitching around the mouth and irritability for last five years. Other physical examination revealed short stature for his age, round face and short neck with no other somatic features. Neurological examination shows no neurological deficit except for positive Chvostik's signs and positive Trousseau's signs. No H/O hypertension, diabetets millitus, asthma, tuberculosis,surgery.Patient is also non-smoker and non alchoholic

On examination patient was alert and oriented. No pallor, clubbing, cyanosis, edema, jaundice.

PR- 78/min, BP- 126/80 mmHg, oxygen saturation- 98\%(without oxygen).

Chest- B/L clear, CVS- $\mathrm{S}_{1} \mathrm{~S}_{2}(+)$, P/A- Soft, B/S(+), CNS- Within normal limit.

\section{Investigations}

S.Calcium-4.6mg/dl(nomal-8.5-10.6),S.Phophorus-7.6mg/dl(normal-2.5-4.5),PTH-186pg/ml(normal15-65) NCCT brain shows multiple coarse calcification in bilateral basal ganglia and deep white matter. Skeletal survey revealed no significant abnormality except calvaral thickening with increased diploi space noted with decrease density. All other routine investigations are within normal limits.

\section{Discussion}

PHP is a rare genetic disorder characterised by hypocalcaemia and hyperphosphataemia secondary to PTH insensitivity.A case report study by Pui Lin Chong, Darryl R Meeking found that a prevalence of 3.4/million. ${ }^{3}$

The molecular defect lies in the GNAS gene encoding the $\alpha$-subunit of the stimulatory G protein $(\mathrm{Gs} \alpha)$. Several variants have been identified. ${ }^{4}$ The following discussion is being focus on the two commonly described subtypes of PHP i.e type 1-PHPla and PHPlb. In PHP-la there is hypocalcemia, hyperphoshatemia, response of urinary cAMP to exogenous PTH is decreased, S.PTH is elevated, stimulatory G alpha subunit dificient, Albright's hereditary osteodystrophy is present and resistance to hormones other than PTH may aslo be present. In PHP-lb there is hypocalcemia, hyperphosphatemia, response of urinary cAMP to PTH is low, S.PTH is elevated, stimulatory G alpha subunit is normal, AHO is absent, resistance to hormones other than PTH is present in some patients .This patient was diagnosed to have PHP type 1a, on the basis of somatic features of AHO and biochemical abnormalities. ${ }^{5}$

[1]. Chong PL et al. Am J Med Sci 2013;10(1):2012-5

\section{References:}

[2]. Mannava P, Masood A, Devi KA. Ind J Biochem Jan-March 2015;30(1):113-16

[3]. Nakamura Y, Matsumoto T, Tamakoshi A, et al. Prevalance of idiopathic hypoparathyroidism and pseudohypoparathyroidism in Japan.J Epidemiol 2000,10:29-33 
[4]. Mantovani G. Clinical review: psuedohypoparathyroidism: diagnosis and treatment. J Clin Endocrinol Metab 2011;96:3020-30

[5]. Chen LP,Chen LH,Wu DA. Journal of Endocrinology and Metabolism 2010;7(2):36-8 